\title{
Effect of Planting Density on The Landscaping Potentials of Pelargonium zonale L. and Santolina chamaecyparissus L. Plants
}

\author{
Nooh, A. ${ }^{1}$, Khattab,M. ${ }^{1}$, Koreish,E. ${ }^{2}$ and R.El-Tanbouly ${ }^{1}$
}

\begin{abstract}
The present work was carried out in the Flower and Ornamental Plants Research Gardens, Faculty of Agriculture Alexandria University, Egypt during the years of 2010 and 2011. Two local cultivars of of Perlgonium zonale L. and Santolina chamaecyparissus $\mathrm{L}$. were used in this work to study the effect of planting density on the parameters that affected their potential landscape uses.

Different planting density were used i.e. 6, 9, 12 and 15 plants $/ \mathrm{m}^{2}$ for Pelargonium and $9,12,15$ and 18 plants $/ \mathrm{m}^{2}$ for Santolina.

Results indicated that increasing the planting density (plants $/ \mathrm{m}^{2}$ ) significantly increased only plant height. However, it decreased the plant diameter, leaf area, number of main branches/plant and slightly decreased plant condition (appearance, health, beauty, ornamental value and function) for Pelarganium and Santolina plants as well.

From the previous results it is recommended to use Pelargonium plants with an average diameter of $28 \mathrm{~cm}$ and at a rate of 15 plants $/ \mathrm{m}^{2}$, while for Santolina plant it is needed to start with a suitable plant (its diameter $>15 \mathrm{~cm}$ ) at rate between 15- 18 plants $/ \mathrm{m}^{2}$ to obtain good landscaping potential and ornamental value.
\end{abstract}

\section{INTRODUCTION}

Landscaping play an important role in delivering good for development. It provides shade, color and softens the appearance of built structures as well as buffering the adverse impacts of development.

Geranium plant (Pelorgonium zonale, L., Family Geraniaceae) is one of the famous and favorite ornamental plants originated in South Africa, then introduced to Europe. It is now widely spread throughout the temperate and subtropical regions of the world (Clifford, 1972). It's an erect perennial, having fibrous roots, leaves are simple nearly round in shape venation is palmate, characterized by horseshoe-shaped dark mark, having scent glands.

The flower color ranges from rose-pink to all shades of red as well as pure white. The distinctly irregular flowers are found in a typically umbel-like inflorescence.

\footnotetext{
${ }^{1}$ Floriculture, ornamental horticulture and landscape gardening dept.

Fac. of Agric. - Elshatby - Alex. Univ. Egypt.

${ }^{2}$ Soil and water sciences dept. Fac. of Agric. - Elshatby -

Alex. Univ. Egypt.

Received December 31, 2014, Accepted February 26, 2015
}

Pelargomium is used in the garden in different positions in beds, around trees, along pathways and walk edgings, on lawns, in pots, in window boxes and wall gardens. Pelargonium can be mixed with other plants in beds and borders.

Santolina plant (Santolina chaecyparissus,L., Family Compositae "Asteraceae") native to southern Europe and Mediterranean region then introduced to European as herb gardens. It's an erect woody herb, leaves are simple minute covering the branches. It is famously known as an aromatic herb and because of its gray color its used for writing on lawns or as a border(Osbrone, 1972).

A lot of people are unaware of the ideal plant spacing for landscape plants for the perfect aesthetic appearance. Also every plant requires space in which to develop normally. The result of close planting is eventually an overcrowded condition and a lack of healthy, well-developed foliage, flower and fruit. The more vigorous specimens crowed out the weaker ones and useless a "thinning-out" process is adopted, the mass effect becomes quite uneven and ragged (Taylor, 1921).

When planting perennial groups some factors must be considered; color, height, blooming period, environmental and culture requirements.

The most important consideration in selecting plant for a perennial garden is to group them according to their environmental and cultural requirements.

The aim of the present work was to reach the suitable planting density for both Pelargonium and Santolina plants.

\section{MATERIALS AND METHODS}

The present work was carried out in the Flower and Ornamental Plants Research Gardens, Faculty of Agriculture, Alexandria university, Egypt during the years 2010 and 2011 .

The experiment was carried out to study the effect of the plant density of Pelargonium zonale and Santolina chamaecyparissus L. on their landscaping potentials.

The cuttings of both plants were taken from the mother plants in uniform length with an average of 15 
$\mathrm{cm}$ and then planted in $50 \mathrm{~cm}$ diameter pots (50 cuttings per pot) in well aerated soil ( 2 sand: 1 clay) and placed in a shade place on November 2010.

One month after planting the rooted cuttings were transplanted to $15 \mathrm{~cm}$ pots filled with an equal mixture of sand and clay soil.

After another month from transplanting, the plants were cultivated into flowerbeds with one square meter area and each flower bed contained different number of the used plants (planting densities).

The plants were fertilized with balanced complete fertilizers (one gram per liter, $500 \mathrm{ml}$ for each pot weekly), a complete fertilizer (20-20-20) was used for Pelargonium plants (Siktberg, 2006), and (20-10-20) was used for Santolina plants (Blessington et al.,2005).

Soil samples were collected at a depth ranged between $0-15 \mathrm{~cm}$ before cultivation for the chemical analysis.

The soil $\mathrm{pH}$ and electric conductivity were 7.89 and $14.8 \mathrm{ds} / \mathrm{m}$, respectively.

As for the soluble cations the potassium, sodium, magnesium and calcium were 1.9, 91.1, 51.5 and 28.5 meq/l, respectively, while the soluble anions of $\mathrm{Cl}^{-}$and $\mathrm{HCO}_{3}$ were 130.3 and 7.5 meq./1, respectively.

Four planting densities were used separately for each plant, namely $6,9,12$ and 15 plants $/ \mathrm{m} 2$ for pelargonium, and $9,12,15$ and 18 plants $/ \mathrm{m}^{2}$ for santolina.

The experiment design was randomized complete block design with three replicates and each replicate contained four treatments for each plant. (Snedecor and Cochran,1974).

The experiment continued till October 2011 and the following data were recorded in both experiments:

Plant height, plant diameter, cover percentage, stem diameter, number of main branches, number of leaves per plants (for Pelargonium only), leaf area (for Pelargonium only), number of inflorescences per plant (only for Pelargonium), flowering date (days) (for Pelargonium only), growth shape (five grades), plant condition (five categories from 9 to 1), flowering intensity (for Pelargonium with grades from 9 to 1 ), flower quality (for Pelargonium with 9 grades from 9 to 1) and the chemical soil analysis was done too at the end of the experiments.

Note: cover percentage, growth shape, plant condition, flower intensity and flower quality were done as reported by Nooh, 1981.

\section{RESULTS AND DISCUSSION}

The results(in tables from 1 to 18 ) indicated that increasing the planting density (plants $/ \mathrm{m}^{2}$ ) significantly increased only plant height. However, it decreased plant diameter, cover percentage, leaf area, number of main branches/plant and slightly decreased plant condition (appearance, health, beauty, ornamental value and function) for the two used plants Pelargonium and Santolina. Furthermore, some of the plants characteristics (i.e. stem diameter, number of leaves/plant and number of inflorescences /plant) were significantly affected.

Besides, results showed that after 8 months from the starting of the experiments using Pelargonium at 15 plants $/ \mathrm{m}^{2}$ covered $91 \%$ of area, while cultivation Santolina at 18 plants $/ \mathrm{m}^{2}$ covered only $32 \%$ of area.

These results were due to the use of small sized Santolina plants at the beginning of the experiment, which they were not enough to reach their maturity size during 8 months and cover a suitable area.

Also, suitable planting density increases the landscape value of ornamental plants where they perform normal growth shape.

Increasing planting density lead to growth competition for achieving more spacing, which inhibits underground growth but enhances the stem growth (Degenhardt and Kondra, 1981, Rao et al.,1989; Morrisson et al.,1990)

The highest flower number/plant was obtained in the lowest plant density.

These results may be attributed to increase the competition between cultivated plants for nutrition and light, which led to form many branches per plant, consequently many flowers could be formed and development per plant.

The progressive increases in plant height and internodes length at decreasing plant spacing and the corresponding decreases in stem diameter (to a greater extent inside compared to outside plants) indicate diminishing carbohydrate suppliers (Kaname and Itagi 1970; Zahara and Timm 1973; Crothers and westermann 1976) appearently because of decreasing plant exposure to light and have been associated with significant reductions in photosynthetic productivity (Papadopoulos and Ormrod 1988).

Generally, the greater the population density, the more competition there will be for resources. Through this population, one or more strained resources will become limiting for plant growth. As population density increases, the growth of individual plants will be limited.

Most researchers found that planting density affects the plant growth, Nooh(1981) on some annuals, 
perennials and woody plants, Roychowdhurg (1989) on Gladiolus plants, Shrivastava and Pahapalker (1997) on Asparagus racemosus, Korikanthimath et al.(1998) on Elettaria cardamomum, Khattak et al.(2004) on Dendranthema grandiflorum, Sadeghi (2009) on Ocimum basilicum and Berimavardi et al. (2011) on Calendula officinals.

Data in Table 19 illustrated the chemical analysis of the soil at the end of the growing season. Showing that there was a slight difference between soil analysis at the beginning of the experiment and at its end. The soil electric conductivity decreased from $14.8 \mathrm{ds} / \mathrm{m}$ to 10.8 $\mathrm{ds} / \mathrm{m}$. Also the content of the flowerbed soil at the analyzed cations was decreased with one exception of calcium which was increased.

For the soluble anions content of the flowerbed soil the chloride and bicarbonate content were increased from 130.3 to 148 meq./1 and 7.5 meq./1 to 15 meq./1 respectively.

The changes between the values of the chemical analysis of the used flowerbeds at the beginning and at the end of the experiment were probably due to many factors such as irrigation water, fertilization, capability of plants for ions absorption, and other environmental conditions.

Table 1. Means of height $(\mathrm{cm})$ of Pelargonium zonale $L$. as affected by different planting density treatments. (plants $/ \mathrm{m}^{2}$ )

\begin{tabular}{lccccccc}
\hline $\begin{array}{l}\text { Treatments } \\
\left(\mathbf{p l a n t} / \mathbf{m}^{\mathbf{2}}\right)\end{array}$ & May & June & July & August & September & October & Means \\
\cline { 2 - 8 } & 16.20 & 18.06 & 19.13 & 20.40 & 21.93 & 23.80 & 19.92 \\
\hline 9 & 16.80 & 18.20 & 19.60 & 22.06 & 25.53 & 28.47 & 21.78 \\
\hline 12 & 16.80 & 18.06 & 20.47 & 23.67 & 27.47 & 31.73 & 23.03 \\
\hline 15 & 16.60 & 17.90 & 21.13 & 23.06 & 30.20 & 37.07 & 24.42 \\
\hline L.S.D at 0.05 & N.S. & N.S. & N.S. & 2.28 & 4.19 & 6.36 & \\
\hline
\end{tabular}

Table 2. Means of plant diameter $(\mathrm{cm})$ of Pelargonium zonale $\mathrm{L}$. as affected by different planting density treatments. (plants $/ \mathbf{m}^{2}$ )

\begin{tabular}{lccccccc}
\hline $\begin{array}{l}\text { Treatments } \\
\left(\text { plant } / \mathbf{m}^{\mathbf{2}}\right)\end{array}$ & May & June & July & August & September & October & Means \\
\hline & 10.27 & 15.20 & 19.03 & 25.43 & 30.30 & 35.20 & 22.62 \\
\hline 6 & 8.67 & 14.73 & 17.87 & 21.76 & 27.80 & 32.97 & 20.63 \\
\hline 12 & 8.93 & 14.20 & 17.26 & 21.70 & 25.57 & 28.93 & 20.46 \\
\hline 15 & 8.67 & 14.53 & 18.16 & 20.50 & 23.73 & 27.77 & 19.24 \\
\hline L.S.D at 0.05 & N.S. & N.S. & N.S. & 2.849 & 4.793 & 3.82 & \\
\hline
\end{tabular}

Table 3. Means of cover percentage (\%) of Pelargonium zonale $\mathrm{L}$. as affected by different planting density treatments. (plants $/ \mathbf{m}^{2}$ )

\begin{tabular}{lccccccc}
\hline $\begin{array}{l}\text { Treatments } \\
\left(\text { plant } / \mathbf{m}^{\mathbf{2}}\right)\end{array}$ & May & June & July & August & September & October & Means \\
\hline 6 & 4.96 & 11.00 & 17.13 & 30.57 & 43.30 & 58.80 & 27.63 \\
\hline 9 & 5.33 & 15.37 & 22.57 & 33.6 & 55.07 & 76.70 & 34.77 \\
\hline 12 & 7.57 & 19.00 & 28.10 & 44.3 & 62.00 & 78.97 & 39.99 \\
\hline 15 & 8.83 & 24.87 & 38.83 & 49.47 & 66.30 & 90.90 & 46.53 \\
\hline L.S.D at 0.05 & 2.06 & 2.77 & 3.89 & 8.53 & 20.97 & 14.00 & \\
\hline
\end{tabular}

Table 4. Means of stem diameter $(\mathrm{mm})$ of Pelargonium zonale $L$. as affected by different planting density treatments. (plants $/ \mathrm{m}^{2}$ )

\begin{tabular}{lccccccc}
\hline $\begin{array}{l}\text { Treatments } \\
\left.\text { (plant/m } \mathbf{m}^{\mathbf{2}}\right)\end{array}$ & May & June & July & August & September & October & Means \\
\hline 6 & 4.80 & 6.27 & 7.27 & 8.07 & 8.87 & 9.67 & 7.49 \\
\hline 9 & 4.53 & 6.00 & 6.80 & 7.73 & 8.67 & 9.47 & 7.20 \\
\hline 12 & 4.53 & 7.07 & 7.07 & 7.60 & 8.13 & 9.07 & 7.24 \\
\hline 15 & 4.73 & 6.67 & 6.67 & 7.80 & 8.67 & 9.47 & 7.33 \\
\hline L.S.D at 0.05 & N.S. & N.S. & N.S. & N.S. & N.S. & N.S. & \\
\hline
\end{tabular}


Table 5. Means of number of main braanches per plant of Pelargonium zonale L. as affected by different planting density treatments. (plants $/ \mathbf{m}^{2}$ )

\begin{tabular}{lccccccc}
\hline $\begin{array}{l}\text { Treatments } \\
\left(\mathbf{p l a n t} / \mathbf{m}^{\mathbf{2}}\right)\end{array}$ & May & June & July & August & September & October & Means \\
\hline 6 & 0.07 & 0.3 & 1.5 & 2.9 & 4.1 & 5.2 & 2.35 \\
\hline 9 & 0.13 & 0.5 & 1.7 & 2.8 & 3.9 & 5.1 & 2.36 \\
\hline 12 & 0.07 & 0.4 & 1.3 & 2.6 & 3.5 & 4.5 & 2.06 \\
\hline 15 & 0.07 & 0.5 & 1.6 & 2.7 & 3.7 & 4.4 & 2.16 \\
\hline L.S.D at 0.05 & N.S. & N.S. & N.S. & N.S. & N.S. & N.S. & \\
\hline
\end{tabular}

Table 6. Means of number of leaves per plant of Pelargonium zonale $L$. as affected by different planting density treatments. (plants $/ \mathrm{m}^{2}$ )

\begin{tabular}{lccccccc}
\hline $\begin{array}{l}\text { Treatments } \\
\left(\mathbf{p l a n t} / \mathbf{m}^{\mathbf{2}}\right)\end{array}$ & May & June & July & August & September & October & Means \\
\hline 6 & 6.07 & 9.00 & 11.87 & 18.67 & 25.80 & 32.40 & 17.32 \\
\hline 9 & 4.80 & 9.73 & 14.13 & 19.60 & 25.13 & 32.07 & 17.58 \\
\hline 12 & 6.20 & 11.20 & 15.47 & 22.53 & 30.60 & 37.87 & 20.64 \\
\hline 15 & 5.93 & 11.00 & 15.33 & 21.13 & 26.27 & 32.20 & 18.64 \\
\hline L.S.D at 0.05 & N.S. & N.S. & 0.20 & 0.13 & N.S. & N.S. & \\
\hline
\end{tabular}

Table 7: Means of number of inflorescence per plant of Pelargonium zonale $\mathrm{L}$. as affected by different planting density treatments. $\left(\right.$ plants $\left./ \mathbf{m}^{2}\right)$.

\begin{tabular}{lccccccc}
\hline $\begin{array}{l}\text { Treatments } \\
\left(\mathbf{p l a n t} / \mathbf{m}^{\mathbf{2}}\right)\end{array}$ & May & June & July & August & September & October & Means \\
\hline 6 & 0 & 0.53 & 1.27 & 1.33 & 2.07 & 3.07 & 1.65 \\
\hline 9 & 0 & 0.53 & 1.33 & 1.60 & 1.27 & 2.07 & 1.36 \\
\hline 12 & 0 & 0.60 & 0.93 & 0.93 & 1.53 & 2.20 & 1.24 \\
\hline 15 & 0 & 0.53 & 0.80 & 0.93 & 1.20 & 2.07 & 1.11 \\
\hline L.S.D at 0.05 & & N.S. & N.S. & N.S. & N.S. & N.S. & \\
\hline
\end{tabular}

Table 8. Means of plant condition of Pelargonium zonale $\mathrm{L}$. as affected by different planting density treatments. (plants $\left./ \mathbf{m}^{2}\right)$.

\begin{tabular}{lccccccc}
\hline $\begin{array}{l}\text { Treatments } \\
\text { (plant/ } \mathbf{m}^{\mathbf{2}} \text { ) }\end{array}$ & May & June & July & August & September & October & Means \\
\hline 6 & 9.00 & 9.00 & 8.60 & 8.60 & 8.47 & 8.60 & 8.71 \\
\hline 9 & 9.00 & 9.00 & 9.00 & 8.87 & 8.87 & 8.47 & 8.87 \\
\hline 12 & 8.86 & 8.87 & 8.73 & 8.47 & 8.07 & 7.53 & 8.42 \\
\hline 15 & 8.73 & 8.87 & 8.73 & 8.33 & 7.8 & 7.27 & 8.29 \\
\hline L.S.D at 0.05 & N.S. & N.S. & N.S. & N.S. & N.S. & N.S. & \\
\hline
\end{tabular}

Table 9. Means of flower intensity of Pelargonium zonale $\mathrm{L}$. as affected by different planting density treatments. (plants $/ \mathbf{m}^{2}$ )

\begin{tabular}{lccccccc}
\hline $\begin{array}{l}\text { Treatments } \\
\text { (plant } / \mathbf{m}^{2} \text { ) }\end{array}$ & May & June & July & August & September & October & Means \\
\hline 6 & 0 & 6.93 & 6.83 & 6.83 & 6.98 & 7.33 & 6.98 \\
\hline 9 & 0 & 6.43 & 6.73 & 6.88 & 6.83 & 6.83 & 6.74 \\
\hline 12 & 0 & 5.73 & 5.60 & 5.48 & 5.36 & 5.25 & 5.48 \\
\hline 15 & 0 & 5.63 & 5.60 & 5.53 & 5.78 & 5.73 & 5.65 \\
\hline L.S.D at 0.05 & & 0.45 & 0.52 & 0.58 & 0.53 & 0.49 & \\
\hline
\end{tabular}


Table 10. Means of flower quality of Pelargonium zonale L. as affected by different planting density treatments. $\left(\right.$ plants $\left./ \mathbf{m}^{2}\right)$

\begin{tabular}{lccccccc}
\hline Treatments & \multicolumn{7}{c}{ Growing Period } \\
\cline { 2 - 8 }$\left(\mathbf{p l a n t} / \mathbf{m}^{\mathbf{2}}\right)$ & May & June & July & August & September & October & Means \\
\hline 6 & 0 & 7.70 & 7.70 & 7.68 & 7.60 & 7.60 & 7.7 \\
\hline 9 & 0 & 7.10 & 7.73 & 7.55 & 7.33 & 7.17 & 7.4 \\
\hline 12 & 0 & 6.40 & 6.43 & 6.26 & 6.00 & 5.97 & 6.2 \\
\hline 15 & 0 & 6.10 & 6.10 & 5.80 & 5.80 & 5.87 & 5.9 \\
\hline L.S.D at 0.05 & & 0.84 & 0.29 & 0.37 & 0.60 & 0.49 & \\
\hline
\end{tabular}

Table 11. Means of growth shape of Pelargonium zonale $\mathrm{L}$. as affected by different planting density treatments. (plants $/ \mathrm{m}^{2}$ )

\begin{tabular}{lcccccccc}
\hline $\begin{array}{l}\text { Treatments } \\
\left(\text { plant } / \mathbf{m}^{\mathbf{2}}\right)\end{array}$ & May & June & July & August & September & October & Means \\
\hline \multicolumn{1}{c}{} & 0 & 0 & 0 & 0 & 5.93 & 7.10 & 6.5 \\
\hline 9 & 0 & 0 & 0 & 0 & 6.87 & 7.67 & 7.3 \\
\hline 12 & 0 & 0 & 0 & 0 & 6.20 & 7.00 & 6.6 \\
\hline 15 & 0 & 0 & 0 & 0 & 6.33 & 7.67 & 7.0 \\
\hline L.S.D at 0.05 & & & & & N.S. & N.S. &
\end{tabular}

Table 12. Means of plant height (cm.) of Santolina chamaecyparissus L. as affected by different planting density treatments. $\left(\right.$ plants $/ \mathrm{m}^{2}$ )

\begin{tabular}{lccccccc}
\hline $\begin{array}{l}\text { Treatments } \\
\left(\text { plant } / \mathbf{m}^{\mathbf{2}}\right)\end{array}$ & May & June & July & August & September & October & Means \\
\cline { 2 - 8 } 9 & 10.00 & 10.83 & 11.3 & 12.37 & 13.2 & 14.07 & 11.93 \\
\hline 12 & 9.73 & 10.8 & 11.33 & 12.37 & 14.43 & 15.07 & 12.29 \\
\hline 15 & 10.2 & 11.63 & 12.43 & 13.8 & 15.43 & 17.13 & 13.44 \\
\hline 18 & 10.13 & 11.6 & 12.8 & 14.8 & 16.8 & 18.33 & 14.08 \\
\hline L.S.D at 0.05 & N.S. & N.S. & N.S. & 1.64 & 1.20 & 2.00 & \\
\hline
\end{tabular}

Table 13. Means of plant diameter (cm) of Santolina chamaecyparissus L. as affected by different planting density treatments. (plants $/ \mathrm{m}^{2}$ )

\begin{tabular}{lccccccc}
\hline $\begin{array}{l}\text { Treatments } \\
\left(\text { plant} / \mathbf{m}^{2}\right)\end{array}$ & May & June & July & August & September & October & Means \\
\hline 9 & 4.28 & 10.63 & 12.82 & 15.80 & 18.23 & 20.03 & 13.63 \\
\hline 12 & 3.87 & 9.33 & 11.93 & 13.83 & 16.13 & 18.37 & 12.24 \\
\hline 15 & 4.15 & 9.70 & 11.67 & 13.67 & 15.67 & 16.7 & 11.93 \\
\hline 18 & 4.18 & 8.53 & 10.81 & 11.90 & 13.53 & 15.07 & 10.67 \\
\hline L.S.D at 0.05 & N.S. & 0.84 & 1.00 & 1.34 & 0.98 & 1.33 \\
\hline
\end{tabular}

Table 14. Cover percentage (\%) of Santolina chamaecyparissus L. as affected by different planting density treatments. (plants $/ \mathbf{m}^{2}$ )

\begin{tabular}{lccccccc} 
Treatments & \multicolumn{7}{c}{ Growing Period } \\
\cline { 2 - 8 }$\left(\mathbf{p l a n t} / \mathbf{m}^{\mathbf{2}}\right)$ & May & June & July & August & September & October & Means \\
\hline 9 & 1.29 & 8.03 & 11.67 & 17.63 & 23.43 & 28.37 & 15.1 \\
\hline 12 & 1.41 & 8.20 & 13.47 & 18.00 & 24.53 & 31.77 & 16.2 \\
\hline 15 & 2.03 & 11.08 & 16.04 & 22.03 & 25.80 & 32.77 & 18.3 \\
\hline 18 & 2.47 & 10.28 & 16.51 & 20.06 & 28.87 & 32.06 & 18.4 \\
\hline L.S.D at 0.05 & 0.69 & 1.52 & 3.49 & 3.83 & 3.04 & 4.29 & \\
\hline
\end{tabular}


Table 15. Means of stem diameter (mm.) of Santolina chamaecyparissus $\mathrm{L}$. as affected by different planting density treatments. (plants $\left./ \mathbf{m}^{2}\right)$

\begin{tabular}{lccccccc}
\hline $\begin{array}{l}\text { Treatments } \\
\left(\mathbf{p l a n t} / \mathbf{m}^{\mathbf{2}}\right)\end{array}$ & May & June & July & August & September & October & Means \\
\hline 9 & 2.93 & 3.40 & 3.40 & 4.13 & 4.33 & 4.33 & 3.75 \\
\hline 12 & 3.00 & 3.26 & 3.27 & 3.87 & 3.93 & 3.93 & 3.54 \\
\hline 15 & 2.93 & 3.67 & 3.67 & 4.06 & 4.13 & 4.13 & 3.76 \\
\hline 18 & 2.93 & 3.60 & 3.60 & 3.67 & 3.93 & 4.00 & 3.62 \\
\hline L.S.D at 0.05 & N.S & N.S & N.S & N.S & N.S & N.S & \\
\hline
\end{tabular}

Table 16. Means of number main of branches per plant of Santolina chamaecyparissus $\mathrm{L}$. as affected by different planting density treatments. (plants $/ \mathrm{m}^{2}$ )

\begin{tabular}{lccccccc}
\hline $\begin{array}{l}\text { Treatments } \\
\left(\mathbf{p l a n t} / \mathbf{m}^{\mathbf{2}}\right)\end{array}$ & May & June & July & August & September & October & Means \\
\cline { 2 - 8 } 9 & 1.87 & 4.00 & 7.87 & 10.93 & 15.43 & 20.83 & 10.15 \\
\hline 12 & 2.00 & 3.93 & 7.60 & 10.60 & 13.67 & 14.33 & 8.69 \\
\hline 15 & 1.87 & 3.80 & 7.47 & 10.47 & 13.03 & 14.13 & 8.46 \\
\hline 18 & 2.00 & 4.00 & 7.03 & 10.07 & 11.47 & 12.00 & 7.08 \\
\hline L.S.D at 0.05 & N.S & N.S & N.S & N.S & 0.91 & 2.12 & \\
\hline
\end{tabular}

Table 17. Means of plant condition of Santolina chamaecyparissus $L$. as affected by different planting density treatments. (plants $/ \mathrm{m}^{2}$ )

\begin{tabular}{lccccccc}
\hline $\begin{array}{l}\text { Treatments } \\
\left(\mathbf{p l a n t} / \mathbf{m}^{\mathbf{2}}\right)\end{array}$ & \multicolumn{7}{c}{ Growing Period } \\
\cline { 2 - 8 } & May & June & July & August & September & October & Means \\
\hline 9 & 9.00 & 8.60 & 8.86 & 8.86 & 8.86 & 8.86 & 8.84 \\
\hline 12 & 9.00 & 8.70 & 8.71 & 9.00 & 8.86 & 8.73 & 8.84 \\
\hline 15 & 9.00 & 9.00 & 9.00 & 8.86 & 8.73 & 8.73 & 8.89 \\
\hline 18 & 9.00 & 9.00 & 8.73 & 8.60 & 8.86 & 8.73 & 8.82 \\
\hline L.S.D at 0.05 & N.S & N.S & N.S & N.S & N.S & N.S & \\
\hline
\end{tabular}

Table 18. Means of growth shape of Santolina chamaecyparissus $\mathbf{L}$. as affected by different planting density treatments. (plants $/ \mathbf{m}^{2}$ )

\begin{tabular}{|c|c|c|c|c|c|c|c|}
\hline \multirow{2}{*}{$\begin{array}{l}\text { Treatments } \\
\left(\text { plant } / \mathbf{m}^{2}\right)\end{array}$} & \multicolumn{7}{|c|}{ Growing Period } \\
\hline & May & June & July & August & September & October & Means \\
\hline 9 & 0 & 0 & 0 & 0 & 7.53 & 7.80 & 7.66 \\
\hline 12 & 0 & 0 & 0 & 0 & 8.60 & 8.30 & 8.45 \\
\hline 15 & 0 & 0 & 0 & 0 & 8.06 & 8.70 & 8.38 \\
\hline 18 & 0 & 0 & 0 & 0 & 6.60 & 6.87 & 6.73 \\
\hline L.S.D at 0.05 & & & & & $\mathrm{~N}$. & N.S & \\
\hline
\end{tabular}

N.S = Not Significant at 0.05 level of probability.

L.S.D. $=$ Least significant difference at 0.05 of probability.

Table 19. Soil analysis of Pelargonium zonle L. and Santolina chamaecyparissus L flowerbeds as affected by different planting density treatments. (plants $/ \mathrm{m}^{2}$ )

\begin{tabular}{|c|c|c|c|c|c|c|c|c|c|}
\hline \multirow{3}{*}{$\begin{array}{l}\text { Experiment } \\
\text { type }\end{array}$} & \multicolumn{9}{|c|}{ Soil Characteristics } \\
\hline & \multirow{2}{*}{$\begin{array}{c}\text { EC } \\
\mathrm{dS} / \mathbf{m}\end{array}$} & \multirow[t]{2}{*}{ pH } & \multicolumn{4}{|c|}{ Soluble cations meq./1 } & \multicolumn{2}{|c|}{ Soluble anions meq./1 } & \multirow[t]{2}{*}{ SAR } \\
\hline & & & $\mathrm{Na}^{+}$ & $\mathbf{K}^{+}$ & $\mathbf{M g}^{++}$ & $\mathrm{Ca}^{+}$ & $\mathrm{Cl}^{-}$ & $\mathrm{HCO}_{3}$ & \\
\hline Flowerbeds & 10.8 & 7.41 & 60.8 & 0.9 & 30 & 52.5 & 148 & 15 & 14.7 \\
\hline
\end{tabular}




\section{REFERENCES}

Berimavandi, A.Reza; Davood Hashemabadi; M.V. Facouri Ghazaini and Behzad Kaviani (2011). Effect of plant density and sowing date on the growth, flowering and quantity of essential oil of Calendula officinalis L. Journal of Medicinal Plants Research 5(20): 5110 - 5115.

Blessington, T.M.; Clement, D.L.; Wadkins, S.L., and Williams, K.G. (2005). Gray Santolina Production and Care. Central Maryland Research and Education Center. Ellicott: Maryland Cooperative Extension.

Clifford, D. (1972). Pelargonium including popular "Geranium". Text Book Blandford Press Limited $2^{\text {nd }}$ edition. U.K.

Crothers, S. E. and westermann, D. T. (1976). Plant population effects on the seed yield of Phaseolus vulgaris L. Agron. J. 63: 958-960.

Degenhardt, D.E and Kondra, Z.P. (1981). The influence of seeding date and seeding rate on seed yield and yield components of five genotypes of Brassica napus (J). Canadian Journal of Plant Science, 61: 175 - 183.

Kaname, T. and Itagi, T. (1970). Studies on the effectivenss use of light in the greenhouse cultivation. I. Effects of the shading on the growth of cucumber. Kanagawa Horticultural Experiment Station. Bulletin No. 18: 97-105.

Khattak, A.M. ; S. Pearson and C.B. Johnson (2004). The effects of far red spectral filters and plant density on the growth and development of chrysanthemums. Scientia Horticulturae, 102, $335-341$.

Korikanthimath, V.S; Hegde R.; Mulge R. and Hosmani M.M.(1998). Growth and yield parameters of cardamom ( Elettaria cardamomum Maton) as influenced by nutrition and plant density. Journ.of Spices and Aromatic Crops 7 (1): $39-42$.

Morrison, M J.; McVetty, EB.E. and scarth, R. (1990). Effect of altering plant density on growth characteristics of summer rape (J). Candian Journal of Plant Science, 70:139 -149 .
Nooh, A. (1981). Studies on the initial state of some selected woody and annual plants as a base for the suitable using in the shaping of green areas and landscape. PhD. University of Humbolat (Berlin).

Osborne, R. (1972). How to grow herbs. Text Book, Lane publishing Co.Menlo park, California. $1^{\text {st }}$ edition: 73.

Papadopoulos, A.P. and Ormrod, D. P. (1988). Plant spacing effects on photosynthesis and transpiration of the greenhouse tomato. Can. J.Plant Sci.68: 1209 - 1218.

Rao, R.C.N.; L.P. Simmonds; S.N. Azam-Ali and J. H. Williams (1989). Population, growth, and water use of groundnut maintained on stored water. I. Root and shoot growth (J). Experimental Agriculture, 25:51 - 61.

Roychowdhury, N. (1989). Effect of plant spacing and growth regulators on the growth and flower yield of Gladiolus grown under polythene tunnel. Acta Horticulturae, 264,246: 259.

Sadeghi Sedigheh, A.R. (2009). The effect of plant-density and sowing-date on yield of Basil. Journal of Agricultural Technology, 5(2), 413 - 422.

Shrivastava, J.L. and Pahapalkar, M.K. (1997). Cultivation trials on Satawar Asparagus racemousus in Mandla district of Madhya Pradesh. Vaniki-Sandesh, 21(2): 24 33.

Siktberg, R.(2006). Pelargoniums An Herb Society of America Guide. Retrieved from http://www.herbsociety.org.

Snedecor, G., and W. Cochran (1974). Statistical methods. Sixth edition. Iowa State University Pres.Ames.Iowa, U.S.A.

Taylor, A.D. (1921). The complete garden. Text Book garden city publishing Co., New York. $1^{\text {st }}$ edition: 29.

Zahara, M. and Timm, H. (1973). Influence of plant density on growth, nutrient composition, yield and quality of mechanically harvested tomatoes. Journal of American Soc.Hortic. Sci. 98 : 513 - 516. 


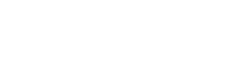

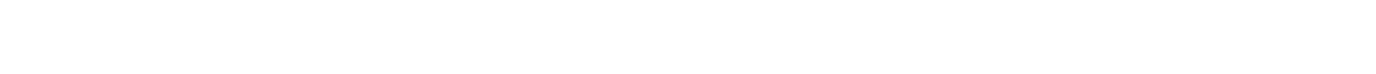

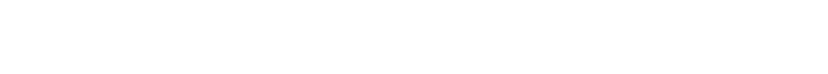

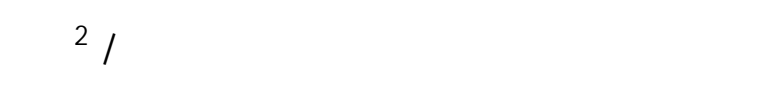

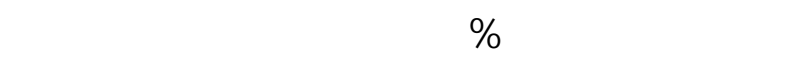

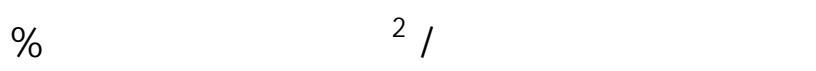

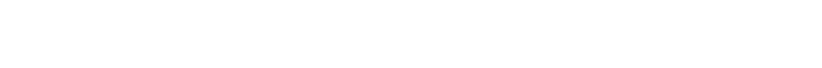
. (a)

وعموما يوص بزراعة الجارونيا العادية بمتوسط قطر

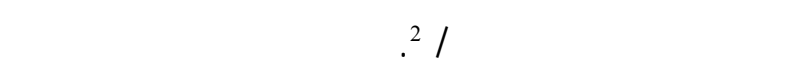

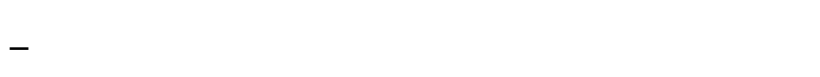

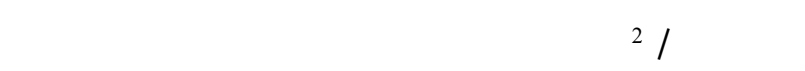
جيلة.
لجرى هذا البهث بحدأق أبحلث الزهور ونباتلت الزينة

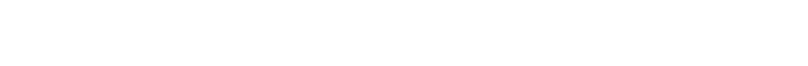

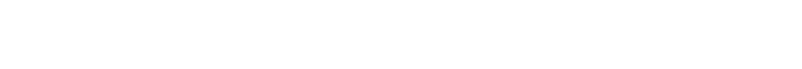

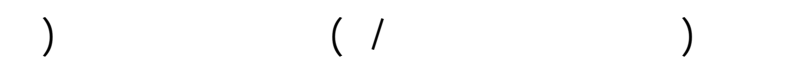

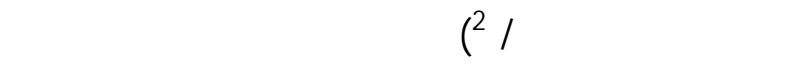
لهما. وقد أظلهرت النتائج المتحصل عليها لكلا النباتين أن الن النيان

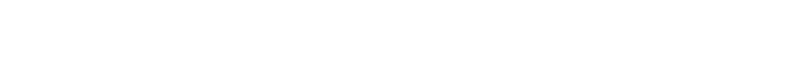

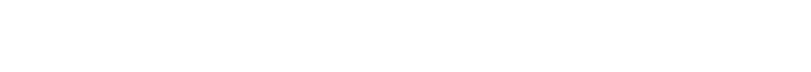

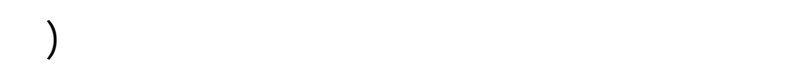
حيث الفظهر وجوة النبلت وقيمته الجمالية وقدرته التنسقية). 